\title{
A RELATIONSHIP BETWEEN THE COMPLETE TOPOLOGY AND THE ORDER TOPOLOGY OF A LATTICE
}

\author{
ARNOLD J. INSEL
}

In this note we establish a necessary and sufficient condition for a lattice to be Hausdorff in its complete topology, namely that every net in the lattice has an order convergent subnet. An immediate consequence of this result is that if a lattice is Hausdorff in its complete topology then the lattice is compact and Hausdorff in its order topology and in fact the two topologies are the same. This last result is a partial resolution of a conjecture offered by this author [1].

I. Definitions and results. By a complete subset $C$ of a lattice $L$ we shall mean a nonempty subset $C$ of $L$ such that for each nonempty subset $S$ of $C, S$ possesses both a supremum $s$ and an infimum $t$ in $L$, and furthermore, both $s$ and $t$ are in $C$.

For any nonempty subset $A$ of a complete lattice $L$ let $A^{Q}$ denote the smallest complete subset of $L$ containing $A$, i.e.,

$$
A^{q}=\bigcap\left\{M: M \supset A \text { and } M_{2}^{*} \text { is a complete subset of } L\right\} .
$$

It is easily seen that $\bigvee A^{q}=\bigvee A$, and $\Lambda A^{q}=\Lambda A$.

For a net $\left\{x_{a}\right\}$ in a lattice $L$ we define $\lim \sup \left\{x_{a}\right\}=\Lambda_{a} \wedge\left\{x_{b}: b \geqq a\right\}$ and $\lim \inf \left\{x_{a}\right\}=\mathrm{V}_{a} \wedge\left\{x_{b}: b \geqq a\right\}$. If $\lim \sup \left\{x_{a}\right\}=\lim \inf \left\{x_{a}\right\}=x$, we say that the net $\left\{x_{a}\right\}$ order converges to $x$. A subset $C$ of $L$ is called order closed if no net in $C$ order converges to a point outside of $C$. The collection of order closed sets comprises the closed sets for some topology for $L$. We call this topology the order topology and shall designate it by $O(L)$. The following results concerning the order topology are known:

1. If a net order converges to a point $x$ in $L$ then the net topologically converges to $x$ with respect to the order topology.

2. If $L$ is compact in its order topology then $L$ is complete.

For any lattice $L$, the smallest topology for $L$ in which the complete subsets of $L$ are closed is called the complete topology for $L$ and is designated by $K(L)$. Several of its interesting properties have been studied by this author [1]. We are concerned with the following results:

1. Every lattice is compact in its complete topology.

2. If a lattice $L$ is Hausdorff in its complete topology then $L$ is com. plete.

Received by the editors June 26, 1963. 
3. $K(L) \subset O(L)$, i.e., every complete subset of $L$ is order closed.

4. If $L$ is complete and $\left\{x_{a}\right\}$ is a net in $L$ which converges to a point $x$ with respect to the complete topology then $\lim \inf \left\{x_{a}\right\} \leqq x$ $\leqq \lim \sup \left\{x_{a}\right\}$.

For any topological space $X$ a net $\left\{x_{a}\right\}$ in $X$ is called a universal net if it is eventually in or eventually outside of any subset of $X$. It is well known that every net has a universal subnet [2]. The following properties concerning universal nets are obvious:

1. A space $X$ is compact iff every universal net in $X$ converges.

2. $X$ is Hausdorff iff no universal net in $X$ converges to more than one point.

\section{A theorem about the complete topology.}

LEMMA 1. Let $\left\{x_{a}\right\}$ be a universal net in a complete lattice $L$ with the complete topology $K(L)$. Then for any $x \in L,\left\{x_{a}\right\}$ converges to $x$ iff $x \in \cap_{a}\left\{x_{b}: b \geqq a\right\}$.

Proor. Suppose that $\left\{x_{a}\right\}$ converges to $x$. Then for any $c$ in the domain of $\left\{x_{a}\right\},\left\{x_{a}\right\}$ is eventually inside the closed set $\left\{x_{b}: b \geqq c\right\}$. We conclude that $x \in\left\{x_{b}: b \geqq c\right\}^{a}$. Hence $x \in \bigcap_{a}\left\{x_{b}: b \geqq a\right\}$.

Now suppose that $\left\{x_{a}\right\}$ does not converge to $x$. Then there exists a complete subset $C$ of $L$ such that $x$ is not in $C$ and $\left\{x_{a}\right\}$ is not eventually in the complement of $C$. Therefore by the universality of $\left\{x_{a}\right\}$, $\left\{x_{a}\right\}$ is eventually in $C$. Hence there exists a $d$ in the domain of $\left\{x_{a}\right\}$ such that $\left\{x_{b}: b \geqq d\right\} \subset C$. Since $C$ is complete, $\left\{x_{b}: b \geqq d\right\}^{a} \subset C$. We conclude that $x \notin \cap_{a}\left\{x_{b}: b \geqq a\right\}$.

Lemma 2. Let $L$ be a complete lattice, let $\left\{x_{a}\right\}$ be a universal net in $L$ and let $A=\bigcap_{a}\left\{x_{b}: b \geqq a\right\}$ ? Then:

1. $\lim \sup \left\{x_{a}\right\}=\mathrm{V} A$.

2. $\lim \inf \left\{x_{a}\right\}=\Lambda A$.

PRoof. For $a$ in the domain of $\left\{x_{a}\right\}$ we shall denote $\vee\left\{x_{b}: b \geqq a\right\}$ by $s_{a}$.

For each $a$ in the domain of $\left\{x_{a}\right\}, A \subset\left\{x_{b}: b \geqq a\right\}^{q}$, and hence $\mathrm{V} A \leqq \mathrm{~V}\left\{x_{b}: b \geqq a\right\}^{a}=s_{a}$. We conclude that $\mathrm{V} A \leqq \Lambda_{a} s_{a}=\lim \sup \left\{x_{a}\right\}$.

Let $c$ be an arbitrary element in the domain of $\left\{x_{a}\right\}$. Then for any $d$ in the domain of $\left\{x_{a}\right\}$ such that $d \geqq c$ we have that $\left\{x_{b}: b \geqq d\right\}$ $C\left\{x_{b}: b \geqq c\right\}$ and hence $s_{d} \in\left\{x_{b}: b \geqq c\right\}$.

For any $a$ in the domain of $\left\{x_{a}\right\}$ there exists a $k$ in the domain of $\left\{x_{a}\right\}$ such that $k \geqq a$ and $k \geqq c$. Then $s_{k} \leqq s_{a}$ and $s_{k} \in\left\{x_{b}: b \geqq c\right\} a$. We conclude that $\lim \sup \left\{x_{a}\right\}=\Lambda_{a} s_{a}=\Lambda\left\{s_{b}: b \geqq c\right\} \in\left\{x_{b}: b \geqq c\right\}$. It follows that $\lim \sup \left\{x_{a}\right\} \in \bigcap_{a}\left\{x_{b}: b \geqq a\right\} a=A$. Hence $\lim \sup \left\{x_{a}\right\}$ $\leqq \mathrm{V} A$, and (1) follows. Statement (2) follows dually. 
THEOREM 3. For any lattice $L$ the following are equivalent:

1. L is Hausdorff in its complete topology.

2. Every universal net in $L$ is order convergent.

3. Every net in $L$ has an order convergent subnet.

Proof. a. (1) implies (2).

If $L$ is Hausdorff in its complete topology then $L$ is complete. Let $\left\{x_{a}\right\}$ be a universal net in $L$. Then by Lemma 1 there exists an element $x$ in $L$ such that $\{x\}=\bigcap_{a}\left\{x_{b}: b \geqq a\right\} a$. Applying Lemma 2 we obtain $\lim \sup \left\{x_{a}\right\}=\mathrm{V}\{x\}=x=\Lambda\{x\}=\lim \inf \left\{x_{a}\right\}$. Therefore $\left\{x_{a}\right\}$ order converges to $x$.

b. (2) implies (3).

This is a direct consequent of the fact that every net has a universal subnet.

c. (3) implies (1).

If every net has an order convergent subnet then $L$ is compact in its order topology and hence $L$ is complete. Let $\left\{x_{a}\right\}$ be a net in $L$ with order convergent subnet $\left\{y_{b}\right\}$. If $\left\{y_{b}\right\}$ order converges to $x$ we have that $\lim \sup \left\{y_{b}\right\}=x=\lim \inf \left\{y_{b}\right\}$. Therefore $\left\{y_{b}\right\}$ converges to one and only one element of $L$ with respect to the complete topology, namely $x$. We conclude that every net has a subnet which converges to at most one point in the complete topology. Therefore $L$ is Hausdorff in its complete topology.

COROLlaRy 4. If a lattice $L$ is Hausdorff in its complete topology then

1. $L$ is compact in its order topology.

2. The order topology and the complete topology for $L$ coincide, i.e., the order closed subsets of $L$ may be described as intersections of finite unions of complete subsets of $L$.

Proof. (1) follows trivially from Theorem 3. Since $K(L) \subset O(L)$, the identity map of $L$ with its order topology onto $L$ with its complete topology is continuous. Since $L$ is compact in its order topology and Hausdorff in its complete topology, the identity map is a homeomorphism.

Theorem 3 and Corollary 4 relate the structures of the complete topology and the order topology of a lattice. We may restate these propositions in terms of the structure of the lattice itself.

Let $x$ and $y$ be distinct elements of a lattice $L$. A finite collection $\mathcal{e}$ of complete subsets of $L$ will be called a complete separation of $x$ and $y$ if $\mathfrak{e}$ covers $L$ and if for each $C \in \mathcal{e}$ if $x \in C$ then $y \notin C$. We shall say that $L$ has the complete separation property if there exists a complete separation of any two distinct elements of $L$. 
Theorem 5. A lattice $L$ has the complete separation property iff $L$ is Hausdorff in its complete topology.

Proof. The family $B$ of finite intersections of complements of complete subsets of $L$ is a base for the open sets of the complete topology $K(L)$. Let $x$ and $y$ be distinct elements of $L$. If $L$ is Hausdorff in its complete topology then there exist disjoint open subsets $U$ and $V$ in $B$ such that $x \in U$ and $y \in V$. Also $U=\bigcap_{i=1}^{m}\left(L-C_{i}\right)$ and $V=\bigcap_{i=m+1}^{n}\left(L-C_{i}\right)$ where $\left\{C_{1}, \cdots, C_{m}, \cdots, C_{n}\right\}=\mathrm{e}$ is a finite collection of complete subsets of $L$. It readily follows that $\mathfrak{e}$ is a complete separation of $x$ and $y$.

If $L$ has the complete separation property and $x$ and $y$ are distinct elements of $L$ then there exists a complete separation $D$ of $x$ and $y$. Define: $U=L-U\{C: C \in D$ and $x \notin C\}$. Similarly define: $V=L$ $-\cup\{C: C \in D$ and $y \notin C\}$. It is easily seen that $U$ and $V$ are disjoint neighborhoods of $x$ and $y$ respectively.

Applying Theorem 5 to Theorem 3 and to Corollary 4 we obtain the following alternate statements.

THEOREM 3 '. For any lattice $L$ the following are equivalent:

1. L has the complete separation property.

2. Every universal net in $L$ is order convergent.

3. Every net in $L$ has an order convergent subnet. then:

COROllary $4^{\prime}$. If a lattice $L$ has the complete separation property

1. $L$ is compact in its order topology.

2. The order topology and the complete topology for L coincide.

Theorem $3^{\prime}$ characterizes those lattices for which all nets have order convergent subnets. Such lattices are both compact and Hausdorff in their order topologies. The question naturally arises as to whether this class of lattices includes all lattices which are compact and Hausdorff in their order topologies. This question remains open.

\section{REFERENCES}

1. Arnold J. Insel, $A$ compact topology for a lattice, Proc. Amer. Math. Soc. 14 (1963), 382-385.

2. John L. Kelley, General topology, Van Nostrand, Princeton, N. J., 1955.

3. B. C. Rennie, Theory of lattices, Foister and Jagg, Cambridge, 1951.

University of California, Berkeley 